\title{
PROPUESTAS PARA EL DESARROLLO SOSTENIBLE DE LA VIVIENDA DE INTERÉS SOCIAL
}

\section{PROPOSALS FOR THE SUSTAINABLE DEVELOPMENT OF SOCIAL HOUSING}

\author{
Intriago-Plaza José ${ }^{1 *}$; Muñoz-Zambrano Shalea²; Hormaza-Muñoz Zaida ${ }^{3}$ \\ 1Estudiante de Postgrado del Máster en Ingeniería Civil de la Universidad Técnica de \\ Manabí, UTM. Portoviejo, Ecuador \\ 2Estudiante de Postgrado del Máster de Trabajo Social de la Universidad Laica Eloy \\ Alfaro de Manabí, ULEAM. Manta, Ecuador \\ ${ }^{3}$ Docente de la Universidad Laica Eloy Alfaro de Manabí, ULEAM. Manta, Ecuador
}

*Correo: renato.intriago.plaza@gmail.com

\begin{abstract}
Resumen
La vivienda es una de las necesidades básicas de las personas y familias independientemente de su condición social o económica, no obstante, un sector importante de la mundial no cuenta con viviendas de calidad. En este sentido, surge como respuesta a esta problemática la vivienda de interés social, que debe ser aplicada bajo criterios de flexibilidad, morfología, infraestructura, espacio, confort y con oportunidades de crecimiento personal y económico para las familias que habiten estos espacios. En el desarrollo de este manuscrito se analizan las experiencias de proyectos de vivienda social aplicados en comunidades con necesidades humanas. Se generan propuestas con los requisitos a considerar en el diseño y ejecución de un proyecto sostenible de viviendas sociales. Se concluye que existen varios modelos de viviendas y conjuntos habitacionales propuestos en proyectos de investigación, por tanto, es necesario analizar su factibilidad en función de los parámetros propuestos para las variables de relación social, tecnología y diseño arquitectónico-urbanístico.
\end{abstract}

Palabras clave: construcción, urbanismo, ciudad, costo, sociedad.

\begin{abstract}
Housing is one of the basic needs of people and families regardless of their social or economic status, however, an important sector of the world does not have quality housing. In this sense, social interest housing emerges as a response to this problem, which must be applied under criteria of flexibility, morphology, infrastructure, space, comfort and with opportunities for personal and economic growth for the families that inhabit these spaces. In the development of this manuscript, the experiences of social housing projects applied in communities with human needs are analyzed. Proposals are generated with the requirements to be considered in the design and execution of a sustainable social housing project. It is concluded that there are several models of housing and housing complexes proposed in research projects, therefore, it is necessary to analyze their feasibility based on the parameters proposed for the variables of social relationship, technology and architectural-urban design.
\end{abstract}

Keywords: construction, urban planning, city, cost, society.

Información del manuscrito:

Fecha de recepción: 22 de agosto de 2019

Fecha de aceptación: 4 de diciembre de 2019

Fecha de publicación: 10 de enero de 2020 


\section{Introducción}

El crecimiento poblacional se ha mantenido en auge permanente durante los últimos años, puesto que en el caso de Latinoamérica la población en 1950 era 69 millones de personas y hasta el 2010 aumentó en un $600 \%$. Esto sugiere que la migración se ha expandido de forma masiva y más aún los movimientos migratorios desde las zonas rurales hacia zonas consideradas urbanas. Según el Banco Interamericano de Desarrollo (BID, 2014), aproximadamente 160 millones de personas habitan en barrios informales, lo cual aumenta el costo de la tierra y por lo tanto, la accesibilidad de un terreno 0 vivienda se reduce para personas de escasos recursos.

Ecuador no es la excepción en la problemática de la vivienda, puesto que la población está en franco aumento y se prevé que para el año 2050 , en este país sudamericano habiten 23 millones de personas (INEC, 2010). Esto conlleva a una mayor demanda habitacional, que evidentemente no ha podido ser resuelta por los gobiernos de turno (Corral, 2011).
Uno de los desafíos de mayor relevancia para la gestión pública en Ecuador es la focalización de la vivienda como un mecanismo para garantizar la accesibilidad el acceso a personas de escasos recursos. Por lo tanto, se deben generar propuestas de vivienda de interés social, apuntando hacia una mejora calidad de vida y al crecimiento sustentable desde la perspectiva social y ambiental (Malpass, 2001).

La gran mayoría de las propuestas arquitectónicas para estratos sociales con ingresos escasos carecen de una estructura de calidad y de integración entre las decisiones planificativas y urbanísticas (ElAnwar \& Aziz, 2014). Por lo tanto, se analizan experiencias, así como los principales retos y fundamentos de la vivienda de interés social. Además, se proponen entornos favorables el desarrollo de la vivienda social mediante construcciones sostenibles.

\section{Experiencias para la vivienda} de interés social

Las propuestas generadas hasta el momento han sido evaluadas en función de su relevancia debido al diseño arquitectónico y la capacidad del proyecto para evolucionar en el 
tiempo acorde a la dinámica social de los beneficiarios del modelo propuesto. A partir de las siguientes variables se definen los parámetros que rigen su funcionalidad (PérezPérez, 2016).

Para la variable relación viviendasociedad se evalúan parámetros como la relación que existe entre la vivienda y la centralidad de la ciudad, con el ambiente laboral y los servicios disponibles. Por su parte, en la relación vivienda-entorno se toman en cuenta la morfología típica, infraestructura y equipamientos comunitarios, medio ambiente $y$ espacios de inclusión $y$ vulnerabilidad. Finamente, la vivienda debe ser construida bajo la armonización de parámetros como forma, espacio, confort y adaptabilidad.

Las urbanizaciones son el conglomerado de viviendas diseñadas y distribuidas en función de la demanda; por lo tanto, deben estar ubicadas en áreas centrales, permitir el acceso a espacios públicos donde se genere el intercambio de productos y servicios. Los factores mencionados previamente favorecen la inclusión a la actividad comercial y productiva para potenciar las condiciones económicas de las personas que habitan una vivienda social.

\subsection{Relación vivienda-} necesidades humanas

Para identificar si una vivienda se encuentra condicionada en un ambiente de pobreza o extrema pobreza deben tomarse en cuenta aspectos de calidad; de los cuales el Estado tiene gran responsabilidad. Se identifican los siguientes puntos en torno a la calidad de un conjunto habitacional: accesibilidad a servicios básicos, fomento a la dependencia económica y garantías para trabajar dignamente, cantidad de personas que habitan en una casa o conjunto habitable y la evolución social y desarrollo urbanístico.

En el caso de las necesidades, debe diferenciarse entre lo que necesita y lo que requiere en concordancia con el contexto y forma de vida de una persona. Por tanto, se asocia la satisfacción personal de las personas con el acceso a una vivienda que cumpla con requisitos de calidad y flexibilidad. Estas necesidades están en constante evolución y dependen en gran medida de las tradiciones y 
costumbres, así como la disponibilidad económica de las personas y familias en general (Leckie, 1989).

\section{Propuestas de un entorno para viviendas sociales}

Las viviendas de carácter social deben diseñarse y construirse en base a ciertas características que la posicionen como un medio evolutivo, participativo, incluyente, productivo y flexible. Pérez-Pérez (2016) menciona las siguientes propuestas para obtener un entorno sostenible en el hábitat de viviendas de interés social: i) fomentar el desarrollo de espacios urbanizados en zonas que tengan acceso tanto a servicios básicos y complementarios y en conexión a las demás áreas de la ciudad. Estos espacios sostenibles brindan facilidades para que las familias que habitan estas viviendas tengan la oportunidad de trabajar y relacionarse con el casco comercial de la ciudad.

ii) Se debe considerar la inclusión de espacios para la interrelación social y zonas verdes, definiendo entornos públicos y privados. La densidad poblacional debe concentrarse en edificaciones de altura baja con un máximo de cinco pisos, para de esta forma dar cabida a viviendas de tipo unifamiliar y multifamiliar en diferentes modalidades.

iii) Las estructuras deben garantizar el crecimiento vertical de forma ilimitada sin alterar la estabilidad de la estructura en aspectos como módulos espaciales y retículas.

iv) Si bien se busca optimizar el uso de recursos para garantizar su disponibilidad con el uso de espacios múltiples, es necesario considerar que se debe buscar el mecanismo para promover la independencia de las diferentes áreas que componen el conjunto habitacional.

\section{Retos de las viviendas de interés social}

La realidad de las necesidades actuales en torno a la vivienda radica en factores como la diversidad y flexibilidad, calidad y gestión integral y articulada. Los hogares hoy se componen de estructuras diversas, tales como unipersonales, monoparentales, biparentales; por consiguiente, se presenta un reto acorde a las diversas necesidades que exige que el esquema de la vivienda sea flexible y adaptable. Los patrones urbanísticos permiten dar el soporte a los requerimientos sociales 
y productivos, considerando una dinámica arquitectónica para conjuntos multifamiliares basada en reglamentos de copropiedad y el hábitat comunitario (Escallón, 2011).

Por otra parte, en países latinoamericanos y en específico en Ecuador, las condiciones ambientales y climatológicas, así como los recientes eventos sísmicos exigen el cumplimiento de altos estándares de calidad. Por lo tanto, debe exigirse el cumplimiento de los requisitos mínimos para la construcción de viviendas. En este sentido, la administración pública lidera la responsabilidad, puesto que desde aquí se establecen los topes para el costo de inversión en la construcción de las viviendas de interés social (Córdova, 2015).

\section{Conclusiones}

La generación de viviendas de interés social debe sustentarse en políticas públicas que garanticen las necesidades comunitarias, sociales y económicas de las personas 0 familias que habitarán estos espacios. Asimismo, promover la construcción no solamente de viviendas, sino de ciudad urbanística sostenible para personas de bajos ingresos o escasos recursos.
Existen modelos de viviendas y conjuntos habitacionales propuestos en diversos estudios, por lo tanto, se debe analizar su factibilidad en función de los parámetros propuestos para las variables de interrelación social, tecnología y diseño arquitectónico.

\section{Bibliografía}

BID. (2014). Urbanización rápida y desarrollo: cumbre de América Latina y China. Nueva York: Banco Interamericano de Desarrollo.

Córdova, M. A. (2015). Transformación de las políticas de vivienda social. El Sistema de Incentivos para la Vivienda en la conformación de cuasi-mercados en Ecuador. Iconos. Revista de Ciencias Sociales, (53), 127149.

Corral, A. (2011). Un techo para mi país" Iniciativa para solucionar el déficit de vivienda popular en América Latina. Quito: Pontificia Universidad Católica. Facultad de Economía.

El-Anwar, O., \& Aziz, T. (2014). Integrated UrbanConstruction Planning Framework for Slum Upgrading Projects (Vol. 140). American Society of Civil Engineers. 
Escallón, C. (2011). La vivienda de interés social en Colombia, principios y retos. Revista de Ingeniería, (35), 55-60.

INEC. (2010). Ecuador en Cifras. Quito: Instituto Nacional de Estadísticas y Censos. Obtenido de: http://ecuadorencifras.gob.ec/ resultados

Leckie, S. (1989). Housing as a human right. Environment and Urbanization, 1(2), 90-108. https://doi.org/10.1177/09562 4788900100210

Malpass, P. (2001). The restructuring of social rented housing in Britain: Demunicipalization and the rise of registered social landlords. European
Journal of Housing Policy, 1(1), 1-16. https://doi.org/10.1080/14616 710110036427

Pérez-Pérez, A. L. (2016). El diseño de la vivienda de interés social. La satisfacción de las necesidades y expectativas del usuario. Revista de Arquitectura, 18(1), 67-75. Doi:10.14718/RevArq.2016.1 8.1.7 